Karama MT Al - Nuaimy BDS, MSc (Lec.)

\section{Gag Problem in Dental Treatment As- sessment and Methods to Control it.}

\author{
Department of Dental Basic Science \\ College of Dentistry, University of Mosul
}

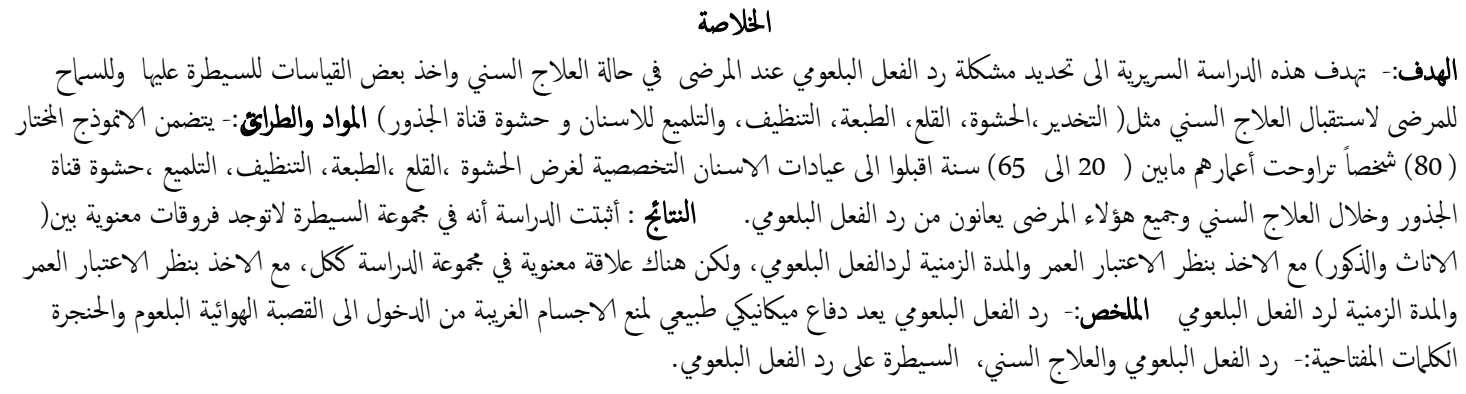

\section{ABSTRACT}

Aims: To assess gag reflex as a problem in patients undergoing dental treatment and some measures used to control it in order to allow the patient to receive dental care such as taking anesthesia, fillings, extractions, impression, scaling and polishing of teeth and root canals. Materials and Methods: The sample chosen included 80 people between the ages 20 and 65 attended to dental clinics for fillings, extractions, impression, scaling and polishing and root canals during dental treatment these patients complained an exaggerated gag reflex. Results: In control group there was no significant difference between females and males regarding the age and duration of gag reflex, but significantly correlated in study group as a whole concerning the age and duration of gag reflex. Conclusions: Gag reflex is considered as normal defense mechanism that prevents foreign bodies from entering the trachea, pharynx or larynx.

Key Words: Gag reflex and dental treatment, control of gag reflex.

Al-Nuaimy KM. Gag Problem in Dental Treatment Assessment and Methods to Control it. AlRafidain Dent J. 2010; 10(2):287-291.

Received: 8/9/2008 Sent to Referees: 8/9/2008

Accepted for Publication:8/6/2009

\section{INTRODUCTION}

Gag reflex is an automatic (involuntary) neuromuscular action elicited by a defined stimulus. It is "triggered" whenever a noxious substance touches the back of the tongue, back of the pharynx or soft palate. ${ }^{(1)}$

The will prevent something from entering the throat. It is also known as a pharyngeal reflex. ${ }^{(2)}$ The normal gag reflex is a mass contraction of both sides of the posterior oral and pharyngeal musculature and an indication by the patient of unpleasant experience. ${ }^{(1,3,4)}$

Many dental patients avoid going to the dentist because of an abnormally severe gag reflex, some of which cannot even brush their back teeth without starting to gag. This is a problem at the dentist clinic since many patients may not be able to withstand having their tongue touched with a dental mirror or other instruments and even figures. It can become an embarrassing and frustrating problem for patients. $^{(1,5)}$

Hyperactive gag reflex is an extreme oro-pharyngeal response to stimulation resulting in pharyngeal and velar contractions occurring for example in divers. ${ }^{(4,6,7)}$

The causes of gag reflex is either somatic (initiated by sensory nerve stimulation from direct contact) or psychogenic (modulated a trigger area induces the reflex). ${ }^{(8)}$

Some people with a bad reflex use an electric tooth brush with a very small brush head, such as the oral -B 3D Excell or professional series. These tooth brushes also do extremely well in controlled studies. ${ }^{(3,4)}$ 
Usually the gag reflex is stronger when brushing back teeth especially the inside and bitting surfaces). Sometime for example the gag reflex may be due to medical problem. ${ }^{(9,10)}$

This study was to evaluate the gag reflex as a problem and methods used to control it, in order to allow the patient to receive dental care such as fillings, extractions, impression, teeth cleaning and root canals with a minimum of anxiety and stress.

\section{MATERIALS AND METHODS}

The sample for the study included 80 people between the ages 20 and 65 , attended to dental clinic for fillings, extractions, impression, teeth cleaning and root canals. During dental treatment these patients complained of an exaggerated gag reflex.

The assessment of gag reflex severity as follows: ${ }^{(1-3,5,8)}$

Grade I (normal gagging reflex), on occasion gagging occurs during high - risk dental procedures such as maxillary impression taking or restoration to the distal, palatal or lingual surfaces of molar teeth.

Grade II (mild gagging), this type of gagging occur occasionally during routine dental procedures such as fillings, scalling and impressions.

Grade III (moderate gagging), this occurs routinely during normal dental procedures. This may include simple physical examination of high risk areas, such as the lingual aspect of lower molars.

Grade IV (severe gagging), it occurs with all forms of dental treatment including simple visual examination.

Grade V (very severe gagging), it occurs easily and may not necessarily, require physical intervension to trigger the reflex.

For patients that are gaggers, put a little topical lidocaine on a cotton tip applicator and put it on the posterior sides of the tongue.

The first way concentrate on breathing through your nose to control gag reflex is breathe, breathe, breathe, say it 3 times because sometimes it is hard to breath through nose.

The using of tablet salt on the tip of the tongue, is also another method, ask the patients to dip their moist finger into a dampen dish of salt and get them to dab it onto the tip of the4ir tongue. Another approach is request from the patient rinse his or her mouth for a few minutes with some normal saline $(0.9 \%$ saline $){ }^{(2,3)}$

The use of nasal decongestant before appointment is very helpful in keeping the nasal passage open to promote breathing through the nose, such as Vicks Ultra Chloraseptic throat spray, which can relieve the gag reflex in gaggers or people with a cough or asthma. Dosage is 2 or 3 sprays right before treatment. It is effect usually lasts up for an hour. ${ }^{(2,8)}$

Some of the popular sedation methods used by patients with severe gag reflex response are:

1. Nitrous oxide.

2. Benzocaine: spray $0.5 \mathrm{mg} / \mathrm{ml}$ can be used to briefly numb the gag reflex areas for simple things like taking a radiograph or taking an impression. The back of the throat stays numb long enough for most people to get through these procedures.

3. Oral sedation with the benzodiazepine medication like valium or halcyon can greatly reduce the gag reflex. ${ }^{(2,3)}$

The local anesthesia can be used in the form of spray, gel, mouth rinse, or injection, by deposition of local anesthetic around the posterior palatine foramen has been used for patients who gag when the posterior palate is touched or by using topical lodicaine $2 \% \mathrm{w} / \mathrm{v}$ on a cotton tip applicator and apply it on the posterior sides of the tongue. ${ }^{(2,8)}$ Hypnosis can also used to reduce gag reflex. ${ }^{(2)}$

\section{RESULTS}

In the study group, the mean age of 45 female and 35 male was 41.3 years $(\mathrm{SD}=$ 19.3; range $20-65$ years) and 42.8 years ( $\mathrm{SD}=17.6$; range $20-65$ years), respectively.

In Table (1), The mean duration of the gag problem among the female and male was 29.8 time/Unit $(\mathrm{SD}=22.3)$ and 32.2 time/Unit $(\mathrm{SD}=21.0)$ respectively. There was no statistical significant difference between females and males with regard to age and duration of the gagging problem. There was significantly correlated in the study group as a whole $(\mathrm{r}=0.94, p=$ 
0.0001) as well as in the female group ( $\mathrm{r}=$ $0.72, p=0.0002)$ and the male group of

Table (1): Numbers of patients distribu patients $(r=0.47, p=0.001)$, concerning the age and duration of the gag problem.

tion

of gag reflex and correlation coefficient in each group.

\begin{tabular}{lccccc}
\hline \multirow{2}{*}{ Number of patients } & Age & Duration of gag reflex & & \multirow{2}{*}{$\boldsymbol{P} *$} \\
\cline { 2 - 3 } Female (45) & Mean \pm SD & Mean \pm SD & & \\
Male (35) & $41.3 \pm 19.3$ & $29.8 \pm 22.3$ & $\mathbf{0 . 7 2}$ & 0.0002 \\
\hline *Significant correlation at $p<0.05$ & $42.8 \pm 17.6$ & $32.2 \pm 21.0$ & 0.47 & 0.001 \\
\hline
\end{tabular}

In Table (2) and (3), before the use of tablet salt 20 patients were unable to accept dental treatment specially filling and scaling, while 32 patients able to accept dental

treatment after using of pharmacological techniques like patients did not accept without using local anesthesia as injection before taking alginate impression.

Table (2): Distribution of patients according to the method of gag reflex control.

\section{Control of gag reflex Number of patient response}

\begin{tabular}{lc}
\hline Pharmaceutical preparation tablet salt & 18 \\
Local anaesthesia (spray or gel) & 30 \\
Local anaesthesia (injection) & 28 \\
Nitrous oxide ointment & 4 \\
\hline
\end{tabular}

Table (3): Distribution of patients according to the Grade of gag reflex

\section{Grade of gag reflex (severity of gag reflex) Number of patient}

\begin{tabular}{lc}
\hline Grade I & 2 \\
Grade II & 16 \\
Grade III & 32 \\
Grade IV & 26 \\
Grade V & 4 \\
\hline
\end{tabular}

\section{DISCUSSION}

The gag reflex is a reflex contraction of the soft palate. It is tested for by touching the back of the patients' pharynx on each side with a spatula. This sensation is mediated via the glossopharyngeal - IXth cranial nerve, while the reflex contraction of the soft palate is mediated via the vagus -Xth cranial nerve.

The gag reflex consider as a common problem faced by dentist during examination or treatment of oral cavity. Every one has gag reflex, if you put your finger down your throat you will provoke it. It also be stimulated from instruments used by the dentist. This can occur in varying degrees, the same patients gagging one or two times throughout dental treatment. ${ }^{(6,8)}$

Relaxation techniques helpful in reducing or abolishing the gag reflex which is done by tense to relax the certain muscle groups, starting with the legs and working upwards the gag reflex is consider a manifestation of an anxiety state..$^{(1,11)}$

Distraction techniques can be used in combination with relaxation procedures, distraction techniques can be useful to temporarily divert procedure to be performed instructed to patients to breathing 
by inhaling through the nose and exhaling through the mouth. ${ }^{(11,12)}$

The most likely cause of a somatically induced gag reflex during dental treatment is the activation of trigger zones in the posterior region of the oral cavity, which are innervated by glossopharyngeal nerve (IX). Those who react with a severe gag reflex when a dental instrument touches the lips or the anterior part of the oral cavity are probably expressing a psychogenically initiated response, as the mucous membrane in the anterior part of the oral cavity is innervated by the trigeminal nerve $(\mathrm{V})$, which is not considered to take part in the gagging reflex, however, there are close connections in the pons between the cranial nerves V, IX and X and abnormal impulse transmission has been described between the vagual and trigeminal nerves. $^{(1,6,13)}$

The one of the main nerves involved in swallowing is the vagus nerve, which also supplies a part of the ear that contains the anti - gagging point. The point is also adjacent to a branch of the trigeminal nerve. Together the trigeminal and the vagus nerves are responsible for much of the sensory and motor functions of the larynx, pharynx and palate. ${ }^{(11,13)}$

The gag reflex has profound impact on the provision of dental care for patients who are affected. The reflex interferes with the ability of the dental practitioner to diagnosis, radiograph and provide treatment for dental patients, and leads to increased levels of stress suffered by patients during treatment sessions. Treatment plans may well be compromised by the need to limit the impact of the reflex, and in some cases unnecessary levels of intervention may be required. ${ }^{(12-14)}$ The reflex can be so trouble some that it in hits patient attendance for treatment and induces anxiety related to dental treatment in certain patients. In severe cases patients actively avoid dental treatment. ${ }^{(6)}$

\section{CONCLUSIONS}

The gag reflex is considered as normal defense mechanism that prevents foreign bodies from entering the trachea, pharynx or larynx. There are many factors which can lead to this problem. Some are obvious and can be eliminated use of alcohol or medication can heighten the gag reflex. The obesity, smoking or tobacco, alcohol or medication can heighten the gag reflex, also dental problems systemic illness and psychological factors like fears can be contributors to eliminated gag reflex.

In general, dental treatment can be performed over a number of visits with reinforcement of the preferred technique at each appointment.

\section{REFERENCES}

1. Saunders RM, Camaron J. Psychogenic gagging: identification and treatment recommendations. Compend. Contin. Educ. Dent. 1997; 18(5): 430 - 433.

2. Murphy WM. A clinical survey of gagging patients. J. Prosthet. Dent. 1997; 42: 145 148.

3. Zach GM. Gag control. Gen. Dent. 1989; 37: $508-509$.

4. Wright SM. The radiologic anatomy of patients who gag with dentures. J. Prosthet. Dent. 1981; 45: 127 - 133.

5. Conny DJ, Tedesc LA. The gagging problem in prosthodontic treatment. Part II: patient management. J. Prosthet. Dent. 1983; 4(6): $757-761$.

6. Humphris GM, Ling ML Behavioral sciences of dentistry. Churchill Livingstone, Edinburgh. 2000. Pp. 73, $81-82$, 88.

7. Andritzky W. Sociopsychotherapeutic functions of a yahuasca healing. Amazonia J. Psychoactive Drugs.2001; 21(1): 77 89.

8. Bassi GS, humphris GM, Longman LP. The etiology and management of gagging. A review of literature. J. Prosthet. Dent. 2004; 91(5): 459 - 467.

9. Safronov VA. Patellar reflex: it associated activation. J. Human Physiology. 1998; 35 -42 .

10. Naylor R, Rudd JA. Emesis and anti emesis. In: Hanks GW (ed). Cancer surveys. Volume 21, Palliative medicine: problem areas in pain and symptom management. New York. Cold. Spring Hanbor Laboratory Press. 1994; 117 - 135.

11. Thayer T. Acupuncture for controlling the gag reflex during upper alginate impression. $2001 ; 1: 481-483$.

12. Hornby PJ. Central neurocircuitry associated with emesis. Am. J. Med. 2001; 111: $106-112$. 


\section{Control of gagging reflex}

13. Fiske J, Dickinson C. The role of acupuncture in controlling the gagging reflex using a review of ten cases. Br. Dent. J. 2001; 190(11): $611-613$.
14. Shanon B. The antipodes of the mindcharting the phenomenology of the ayahuasca experience. Oxford: Oxford University Press. 2002: 78 - 92. 